\title{
Entanglement Generation Based on Quantum Dot Spins
}

\section{Book Chapter}

Author(s):

Delteil, Aymeric; Gao, Wei-bo; Sun, Zhe; Imamoglu, Atac

Publication date:

2017

Permanent link:

https://doi.org/10.3929/ethz-b-000187735

Rights / license:

In Copyright - Non-Commercial Use Permitted

Originally published in:

Nano-Optics and Nanophotonics, https://doi.org/10.1007/978-3-319-56378-7_12 


\title{
Generation of quantum dot spin entanglement
}

A. Delteil, W.-B. Gao, Z. Sun, and A. Imamoğlu

1 Institute of Quantum Electronics, ETH Zurich, 8093 Zurich, Switzerland

2 Div. of Physics and Applied Physics, Nanyang Tech. Univ., Singapore 637371

\begin{abstract}
Quantum correlations between a confined spin and a propagating single photon can be used to entangle distant spins. In this Chapter, we review recent progress in the field culminating in the demonstration of spin-photon entanglement, teleportation of quantum information from a photonic qubit to a quantum dot spin and heralded entanglement of distant hole spins. These results constitute important milestones towards the realization of quantum repeaters and on-chip quantum networks.
\end{abstract}

\subsection{Introduction}

\subsubsection{Motivation}

Quantum networks made of quantum memories interconnected by photons could find applications in long-distance quantum communication based on quantum repeaters as well as in distributed quantum computation [1-4]. Realization of such networks requires a quantum interface between stationary (matter) qubits and flying (photonic) qubits. Self-assembled quantum dots (QDs) feature unique properties that allow for the realization of such an interface:

- they can be deterministically charged with a single electron or hole, the spin of which constitutes a quantum memory of coherence time up to several microseconds, limited by hyperfine interaction [5];

- they exhibit optical transition to excited states (trions) with a short lifetime of about 1 ns. Strong spin-orbit interaction yields spin-dependent selection rules $[6,7]$.

As a consequence, QDs allow for fast initialization [8], manipulation [9] and measurement of the spins using laser excitation. In addition, single photon emission is typically much faster than optical transitions in most other 
matter qubit systems. Moreover, they benefit from the semiconductor technology which allows to design integrated optical and electrical functionalities for instance optical cavities for efficient light extraction [10,12], and diodes for electric field control $[11,12]$ - and open prospects for on-chip integration [13].

In this chapter, we review recent experimental realization of elementary protocols based on self-assembled QDs. We first present the recent demonstration of spin-photon entanglement with QDs (Sect. 1.2), which constitutes the building block for realizing quantum-dot-based quantum networks. We then discuss the indistinguishability of photonic qubits emitted by remote QDs, essential for interconnecting distant nodes (Sect. 1.3). Based on these demonstrations, we review the experimental realization of quantum teleportation from a photonic qubit to a QD spin qubit (Sect. 1.4). We then focus on the recent demonstration of heralded entanglement generation. After having introduced the entanglement generation protocol (Sect. 1.5), we expose a novel method to measure the spin coherence based on interference of inelastic light scattering (Sect. 1.6), followed by the implementation and characterisation of a single qubit phase gate, useful for entanglement verification (Sect. 1.7). In the last part, we discuss the experimental verification of distant entanglement between QD hole spins (Sect. 1.8).

\subsubsection{Quantum dot structures}

The experiments discussed in this chapter are based on InAs/GaAs selfassembled QD samples grown by molecular beam epitaxy (MBE). A single QD layer is embedded in a lossy cavity of $Q \sim 20$, ensuring that a sizeable part of the emitted light will escape the sample in a single free space mode that can be coupled to a fibre. A solid immersion lens (SIL) increases further the collection efficiency, up to a total amount of $\sim 10$ to $20 \%$ depending on the particular structure design. A Schottky (sections 1.2 to 1.4 ) or $p-i-n$ (sections 1.5 to 1.8) diode structure allows to control the charge state of the QD as well as fine tuning of the optical transition energy using quantum confined Stark effect.

\subsection{Quantum dot spin-photon interface}

\subsubsection{Spin-photon entanglement generation scheme}

All demonstrations of entanglement between a photonic qubit and a matter qubit so far are based on excitation to a state external to the qubit subspace, from which radiative decay channels to the qubit states coexist with equal oscillator strength [14-16] (lambda system). Other entanglement generation schemes are in principle possible, for instance using giant Faraday rotation induced by a single spin in a cavity [17], however their experimental realization still remains elusive. 


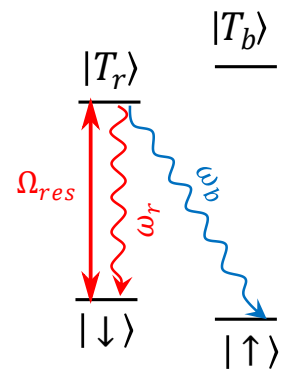

Fig. 1.1. The energy-level diagram of a single-electron-charged InGaAs QD in Voigt geometry. Spontaneous emission following resonant optical excitation of the trion state, $\left|T_{r}\right\rangle$, with Rabi frequency $\Omega_{r e s}$, leads to the generation of an entangled spinphoton state.

In single QD systems, a lambda scheme is formed when applying inplane magnetic field (Voigt geometry), leading to Zeeman splitting of the spin ground states, to which any of the two trion excited states can decay at the same rate. The corresponding energy diagram is shown in Fig. 1.1, as well as the allowed optical transitions from one of the excited states (the red trion). All four transitions between the two ground states and the two excited states have identical oscillator strengths and are linearly polarized [18]. The ground states of the QD are identified by the orientation of the electron spin, with $|\uparrow\rangle$ and $|\downarrow\rangle$ respectively denoting spins parallel and antiparallel to the magnetic field direction. Spontaneous emission of an horizontally $(H)$ or vertically $(V)$ polarized photon at frequency $\omega_{r}$ or, respectively, $\omega_{b}$ from the trion state $\left|T_{r}\right\rangle$ at rate $\Gamma / 2$ brings the QD back into the state $|\downarrow\rangle$ or, respectively, $|\uparrow\rangle$.

The basic principle behind the deterministic generation of a spin-photon entangled state is straightforward: following the excitation of the QD into one of the trion states (here we consider excitation to the red trion), radiative recombination projects the coupled $\mathrm{QD} /$ photonic mode system into the entangled state

$$
|\Psi\rangle=\frac{1}{\sqrt{2}}\left(|\downarrow\rangle\left|\omega_{r} ; H\right\rangle+i|\uparrow\rangle\left|\omega_{b} ; V\right\rangle\right)
$$

written in the computational basis, where $\left|\omega_{r} ; H\right\rangle$ and $\left|\omega_{b} ; V\right\rangle$ denote propagating single-photon-pulse state vectors with respective centre frequencies $\omega_{r}$ and $\omega_{b}$ and respective polarizations $H$ and $V$. The electronic Zeeman energy satisfies $\omega_{Z}=\omega_{b}-\omega_{r}$.

As can be seen on equation 1.1, the spin is entangled with two photonic degrees of freedom: polarization and centre frequency. In order to avoid whichpath information leakage, one of these two photonic degrees of freedom has to be erased to end with a Bell state. Erasure of the centre frequency degree of freedom can be obtained by either frequency down-conversion using short pulses [19] or by time filtering/post-selection using a window shorter 
than the beatnote period [20]. One obtains a state where the spin is entangled with the photon polarization. On the other hand, it is also possible to erase the polarization information using a polarizer at $45^{\circ}$, projecting the photon state on $|H+V\rangle$ or $|H-V\rangle$. This last approach is well suited for fibre-based applications, where the colour degree of freedom is robust to propagation in fibres thanks to a small typical wavelength difference between the two photonic components. In the following we will focus on the latter approach, developed in reference [21], in which the verification of spin-photon entanglement relies on the possibility to perform high-resolution time-resolved resonance fluorescence $[22,23]$.

In reference [21], the spin-photon entangled pair is generated from a singleelectron-charged, self-assembled InGaAs QD, with an external magnetic field of $0.7 \mathrm{~T}$ applied perpendicular to the growth direction.

\subsubsection{Entanglement verification}

Verification of entanglement and estimation of the fidelity to a maximally entangled state need not necessarily a full tomography of the generated state, although the latter can participate to understand fidelity limitations [24]; nonclassical correlations can be evidenced by measuring conditional probabilities in two orthogonal bases [25].

In the case of spin-photon frequency entanglement, the two sets of correlations to be measured are:

- (classical) correlations between the spin in the computational basis and the photon colour

- (quantum) correlations between the spin in a superposition state (r̈otated basis") and the photon in a superposition of the two frequencies

In addition to resonant laser excitation of the trion states, the scheme uses non-resonant, right-hand-circularly $\left(\sigma^{+}\right)$-polarized, 4-ps-long mode-locked laser pulses that are red-detuned from the trion transitions by $\sim 210 \mathrm{GHz}$ : these pulses induce a coherent rotation of the electron spin [9], with minimal trion excitation (see section 1.5.3 of Chap. 10 for a detailed description of this coherent control). The set-up, that integrates two-laser excitation together with time- and frequency-resolved resonance fluorescence, is depicted in Fig. 1.2. Suppression of the $\sigma^{+}$-polarized background laser light is ensured by a polarizer that projects the polarization of the resonance fluorescence photons to $\sigma^{-}$. As mentioned in Sect. 1.2.1, it also erases correlations between the electron spin and the polarization of emitted photons; photonic states in the output mode are then fully characterized by their centre frequency, and entanglement is then demonstrated between the electron spin orientation and the centre frequency of the single-photon pulse.

The pulse sequence we used is depicted on Fig. 1.3a. It consists of: - a 5-ns resonant laser pulse tuned to the $|\downarrow\rangle-\left|T_{r}\right\rangle$ transition, to prepare the QD in state $|\uparrow\rangle$

- a 4-ps $\pi$-pulse that transfers the QD to the $|\downarrow\rangle$ state 


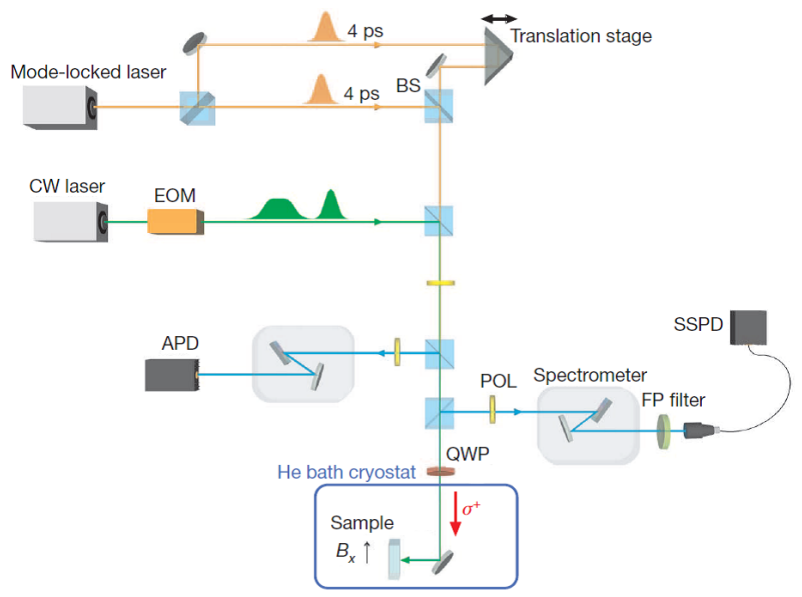

Fig. 1.2. The schematic of the experimental set-up highlighting the important optical elements. BS, beam splitter; CW, continuous wave; EOM, electro-optic modulator, FP, Fabry-Pérot filter; POL, polarizer QWP, quarter-wave plate

- a 1.2-ns resonant laser pulse that generates the spin-photon entangled pair - an additional 4-ps-long spin rotation pulse used to rotate the spinmeasurement basis.

The whole pulse sequence is repeated after $13 \mathrm{~ns}$, with the 5-ns resonant laser implementing spin measurement for the preceding pulse sequence as well as ensuring spin pumping/preparation in the $|\uparrow\rangle$ state for the subsequent cycle. Indeed the detection of a photon at the avalanche photodiode (APD) during this pulse tells with a high confidence level that the spin is in the state $|\downarrow\rangle$. Note that, as the average number of photons emitted during this spin measurement/pumping time window is $\sim 1$ and our combined collection and detection efficiency is $\sim 0.1$, the absence of a detection events brings no information, therefore it implies that we extract heralded spin information [7].

\section{Classical correlations}

Demonstration of spin-photon correlations in the computational basis requires the measurement of classical correlation between the spin direction and the photon centre frequency. This is achieved by carrying out a coincidence measurement between resonance fluorescence photons at either $\left|\omega_{b}\right\rangle$ or $\left|\omega_{r}\right\rangle$ generated immediately after the entanglement pulse and those generated during the measurement/preparation pulse. Conditional on the detection of the spin state $|\downarrow\rangle$, we find that the probability of detecting a blue $\left(\omega_{b}\right)$ photon at the SSPD is drastically suppressed compared with the probability of detecting a red $\left(\omega_{r}\right)$ photon (Fig. 1.3b). With the additional 4-ps $\pi$-pulse introduced at $t=3 \mathrm{~ns}$, it is possible to condition the single-photon colour measurements on 
a

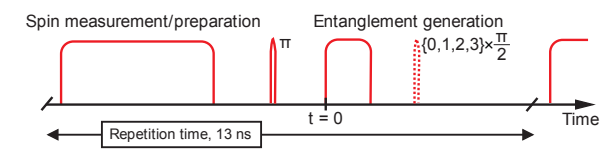

classical correlations:

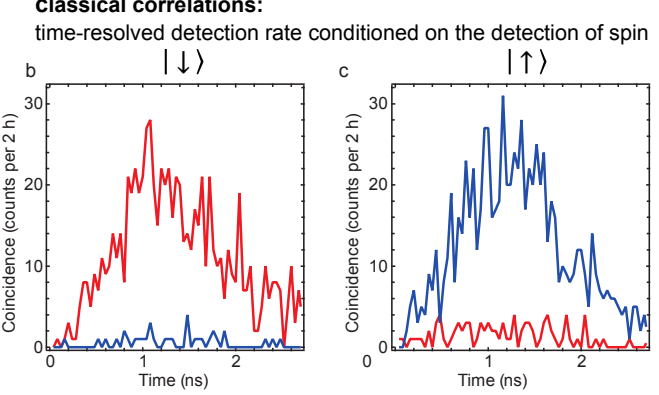

quantum correlations:

time-resolved detection rate conditioned on the detection of spin

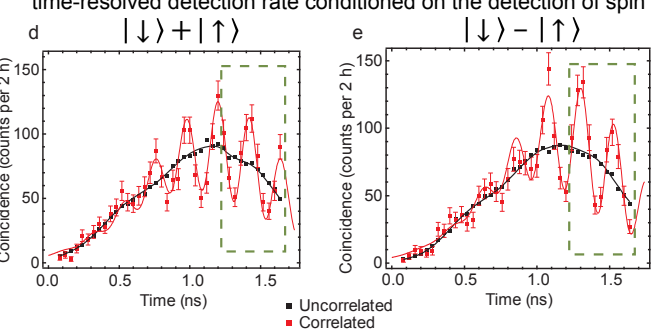

Fig. 1.3. a, pulse sequence used to measure the spin-photon correlations. b, Timedependent resonance fluorescence at $\left|\omega_{r}\right\rangle$ (red) and $\left|\omega_{b}\right\rangle$ (blue), conditioned on detection of a photon during the subsequent measurement/preparation pulse. The strong suppression of conditional photon detection events at $\left|\omega_{b}\right\rangle$ demonstrates the strong classical correlation between spin measurement yielding $|\downarrow\rangle$ and a photon detection at $\left|\omega_{r}\right\rangle$. c, Same as in d but now with a $\pi$-pulse applied before the measurement/preparation pulse, showing strong correlations between spin detection in $|\uparrow\rangle$ and photon detection at $\left|\omega_{b}\right\rangle$. d, Time-resolved coincidence events between the single-photon detection following the entanglement pulse and the detection of a photon during the first measurement/preparation pulse following a $\pi / 2$-pulse (red squares). For comparison, an average of coincidence events between the spin and photon detection events taking place in 25 different excitation/preparation cycles is also shown (black squares). The red curve is a fit to the experimental data. e, same as in d but now with a $3 \pi / 2$-pulse

detection of the spin in $|\uparrow\rangle$. In this case, we find that the red-photon detection events are strongly suppressed (Fig. 1.3c). From these measurements, we determine the fidelity of the classical correlations to be $F_{1}=0.87 \pm 0.09$. 


\section{Quantum correlations}

To verify that the generated spin-photon state is entangled, correlations between spin orientation and photon colour have to be measured in a rotated basis. This verification makes use of the relative phase between the two components of the entangled state in equation 1.1. After generation at time $t_{g}$, the two components acquire a time-dependent phase that stems from the different propagation phase factors of the blue and red frequency components of the photonic mode. In our experiments, we infer the generation time from the detection time, $t_{d}$, of the single photon at the SSPD, through the relation $t_{g}=t_{d}-L / c$, where $L$ denotes the distance from the QD to the detector and $c$ is the speed of light. Hence, given a photon detection event at $t_{d}$, we can a-posteriori write the time dependence of the spin-photon entangled state of equation 1.1 as

$$
|\Psi(t)\rangle=\frac{1}{\sqrt{2}}\left(|\downarrow\rangle\left|\omega_{r} ; H\right\rangle e^{-i \omega_{Z}\left(t-t_{g}\right)}+i|\uparrow\rangle\left|\omega_{b} ; V\right\rangle\right)
$$

The requisite spin measurement is implemented by rotating the QD electron spin with either a $\pi / 2$-pulse or a $3 \pi / 2$ - pulse at $t=t_{1}=1.7 \mathrm{~ns}$. A photon detection event at the APD during the following measurement/preparation pulse then projects the electron spin into $(|\uparrow\rangle-i|\downarrow\rangle) / \sqrt{2}(\pi / 2$-pulse $)$ or $(|\uparrow\rangle+i|\downarrow\rangle) / \sqrt{2}(3 \pi / 2$-pulse). The photonic wavefunction consistent with the spin measurement after the $\pi / 2$-pulse can be written as

$$
\left(\left|\omega_{r} ; H\right\rangle e^{-i \omega_{Z}\left(t-t_{g}\right)}-\left|\omega_{b} ; V\right\rangle\right) / \sqrt{2}
$$

After passing through the polarizer, which fixes the polarization of the photon to be $(|H\rangle-i \mid V) / \sqrt{2}$, this wavefunction is

$$
|\Phi(t)\rangle=\frac{1}{\sqrt{2}}\left(\left|\omega_{r}\right\rangle e^{-i \omega_{Z}\left(t-t_{g}\right)}-i\left|\omega_{b}\right\rangle\right)
$$

The resulting coincidence probability is $P=\left(1+\sin \left(\omega_{Z}\left(t_{1}-t_{g}\right)\right)\right)$. As a consequence of the time-dependent relative phase, two single-photon states generated at different times, $t_{g}^{a}$ and $t_{g}^{b}$, such that $\omega_{Z}\left(t_{g}^{a}-t_{g}^{b}\right)=\pi$, allow us to infer the overlap of the photonic state with two orthogonal states. Random photon detection times together with the fact that the single-photon pulse width, $\tau$, satisfies $\omega_{Z} \tau>1$, effectively implement projective measurements into orthogonal states such as $\left|\omega_{r}\right\rangle \pm\left|\omega_{b}\right\rangle$. The single-photon pulses are measured by a fast superconducting single photon detector (SSPD), which has a jitter of $\Delta \tau=60 \mathrm{ps}$, short enough to resolve the time dependence in equation 1.4.

Figure 1.3b shows the coincidence between the single-photon detection events induced by the entanglement pulse and the detection of a photon during the measurement/preparation pulse, following a $\pi / 2$-pulse: the oscillations 
with a period given by $2 \pi / \omega_{Z}=238$ ps in the conditioned single-photon detection events (Fig. 1.3d, red squares) stems from the conditioned photonic wavefunction given in equation 1.4 and constitute a remarkable manifestation of the quantum coherence of the entangled spin-photon system. For comparison, we also show an average of coincidence events between the spin and photon detection events taking place in different excitation/preparation cycles (Fig. 1.3d, black squares), which do not show any oscillations. Figure 1.3e shows the time-dependent coincidence measurements obtained by applying a $3 \pi / 2$-pulse before the measurement/preparation pulse. Note that the oscillations of visibility $47 \pm 4 \%$ for spin detection along $(|\downarrow\rangle+i|\uparrow\rangle) / \sqrt{2}$ are $\pi$ out of phase relative to those along $(|\downarrow\rangle-i|\uparrow\rangle) / \sqrt{2}$. Using the data from Fig. 1.3d, $\mathrm{e}$, we determine the fidelity of the quantum correlations in this rotated basis to be the average of the two visibilities, yelding $F_{2}=0.46 \pm 0.04$. The overall measured entanglement fidelity is then $F \geq\left(F_{1}+F_{2}\right) / 2=0.67 \pm 0.05$, limited predominantly by the finite jitter of the SSPD.

\subsubsection{Coherence of the entangled pair and spin-photon entanglement with spin-echo sequence}

A major limitation in the demonstration of quantum correlations between the electron spin and the photon frequency stems from the relatively short ground-state spin decoherence time, $T_{2}^{*}$, of the electron spin, originating from the slowly fluctuating nuclear spin environment: owing to the hyperfine interaction between the electron spin and QD nuclear spin ensemble, the electron spin is subject to an effective magnetic field determined by a combination of the fixed externally applied field and the quasi-static random Overhauser field. For different repetitions of the experiment the nuclear spins will be in different states, yielding a corresponding slowly fluctuating Overhauser field and thereby decreasing of the timescale that electron spin coherence is observable. This timescale is commonly referred to as spin $T_{2}^{*}$ dephasing time. We refer the reader to the section 4 of Chap. 9 for an extensive description and characterisation of nuclear-spin-induced electron spin dephasing.

The decay of the oscillations as a function of $t_{1}-t_{g}$ in conditioned singlephoton detection events (Fig. 1.3b, d) constitutes a measurement of the electron spin decoherence that was previously carried out using Ramsey interferometry [5]. By fitting the decay of coincidence measurements in the time window [0 ns, $1.64 \mathrm{~ns}]$ we find that $T_{2}^{*}=1.1 \pm 0.2 \mathrm{~ns}$ for the data in Fig. 1.3b and that $T_{2}^{*}=0.9 \pm 0.2 \mathrm{~ns}$ for the data in Fig. 1.3d, for this QD. The coherence time can be prolonged beyond $T_{2}^{*}$ thanks to a spin-echo sequence, allowing to utilize the spin-photon entangled pair in quantum communication protocols where the network nodes are separated by macroscopic distances. This has been demonstrated in reference [35], where a spin-echo sequence is applied to a singly charged QD as illustrated in Fig. 1.4a, together with the relevant energy-level diagram and the allowed optical transitions under an external magnetic field $B_{x}=0.7 \mathrm{~T}$ that is applied perpendicular to the growth 
direction (Fig. 1.4b, inset). The QD is prepared in $|\downarrow\rangle$ state by applying a 10 ns-long resonant laser pulse tuned to the $|\uparrow\rangle-\left|T_{b}\right\rangle$ transition. A subsequent 4 ps-long $\pi$-pulse ("rotation pulse") transfers the spin onto $|\uparrow\rangle$. An entangled spin-photon pair is then generated by resonant excitation of the $\left|T_{b}\right\rangle$ with a 1.2 ns resonant laser pulse ("entanglement pulse"). The whole pulse sequence is repeated after $104.8 \mathrm{~ns}$, with the subsequent 10-ns long resonant laser implementing spin measurement for the preceding pulse sequence, as well as ensuring spin pumping/preparation in the $|\downarrow\rangle$ state for the next cycle. For an echo time $T_{\text {echo }}=13 \mathrm{~ns}$, a measurement of the spin-photon correlations in a rotated basis is shown in Fig. 1.4b. Combined with measurements that project the photon and the spin in different states (similarly to described in section 1.2.2), we can calculate the spin-photon entanglement fidelity to be $F>0.63 \pm 0.02$. This bound is once again limited by the detection jitter (64 ps). If the detector jitter were absent, we would obtain $F>0.71 \pm 0.02$ $(F>0.82 \pm 0.02)$ as the lower bound for entanglement fidelity with (without) spin-echo.

An alternative way to retain coherence of a spin-photon entangled pair during a longer time is to use hole spins, which exhibit a similar energy level diagram but have a $T_{2}^{*}$ one to two orders of magnitude longer. This is the route taken by the experiments described in sections 1.5 to 1.8 .

Another QD based system that exhibits a lambda scheme which is in principle well suited for generated spin-photon entanglement is a QD molecule in the doubly charged regime, at the so-called sweet spot [26]. Although spinphoton entanglement has not been demonstrated so far, the lambda system formed by the singlet and triplet ground states optically coupled to a common trion state have been used to demonstrate coherent population trapping, showing promising spin coherence of tens to hundreds of nanoseconds.

\subsection{Indistinguishability of photonic qubits emitted by different dots}

The implementations of quantum communication protocols that we will be discussing in the sections 1.4 to 1.8 rely on the indistinguishability of single photons emitted by remote QDs. In this section we show how to generate photonic qubits (that are not entangled with any external degree of freedom) and characterize the indistinguishability of such qubits emitted by two different QDs [35].

\subsubsection{Generation of photonic frequency qubits}

In a neutral self-assembled InGaAs QD, the elementary optical excitations from the unique ground state $|0\rangle$ are the two fundamental exciton states $\left|X_{r}\right\rangle$ and $\left|X_{b}\right\rangle$ that are split in energy by the anisotropic electron-hole exchange interaction [38]. It is therefore possible to generate a photonic qubit 

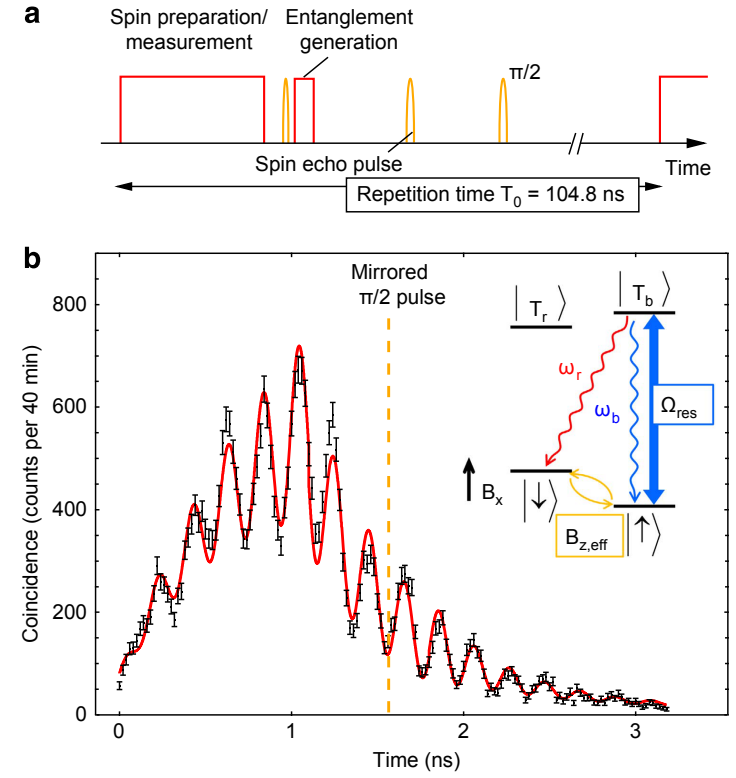

Fig. 1.4. a The laser pulse sequence used for the generation of spin-photon entangled state and measurement of quantum correlations after a spin-echo sequence. $b$ Spin-photon entanglement in the rotated basis and the relevant energy-level diagram (right inset). The main figure shows the photon detection events as a function of time, during and after the entanglement generation pulse, conditioned upon a spin-echo-delayed spin measurement in the $(|\downarrow\rangle+|\uparrow\rangle) / \sqrt{2}$ state. The entanglement generation pulse is turned on at time $t=0$ and is $1.2 \mathrm{~ns}$ long. The oscillations at $\omega_{b}-\omega_{r}=4.9 \mathrm{GHz}$ are due to the beating between the two frequency components of the projected single-photon superposition state. The vertical dashed line indicates the entangled spin-photon generation time for which the spin-echo sequence exactly cancels the random phase accumulated due to hyperfine interactions

(see section 1.1 of Chap. 7) by resonant pulsed excitations of these excitonic transitions. The exciton state $\left|X_{r}\right\rangle\left(\left|X_{b}\right\rangle\right)$ decays at a rate $\Gamma_{1}$ by spontaneous emission of a photon at frequency $\omega_{r}\left(\omega_{b}\right)$ back into $|0\rangle$. A laser pulse resonant with either of these two exciton states results in the generation of a single-colour single photon states denoted by $\left|\omega_{r}\right\rangle,\left|\omega_{b}\right\rangle$. Alternatively, applying a two-colour laser pulse that is simultaneously resonant with both $\left|X_{r}\right\rangle$ and $\left|X_{b}\right\rangle$ results in the superposition state $\alpha\left|\omega_{r}\right\rangle+\beta\left|\omega_{b}\right\rangle$ (left inset of Fig 1.5).

A two-colour laser pulse that excites the QD in such a superposition of exciton states can be obtained by tuning the frequency of a diode laser exactly in the middle of the two neutral exciton transitions and sending the beam to an amplitude electro-optic modulator (EOM) that generates pulses from the continuous-wave laser field. A phase EOM is used to generate laser sidebands that are on resonance with the exciton transitions, and enable us to resonantly 


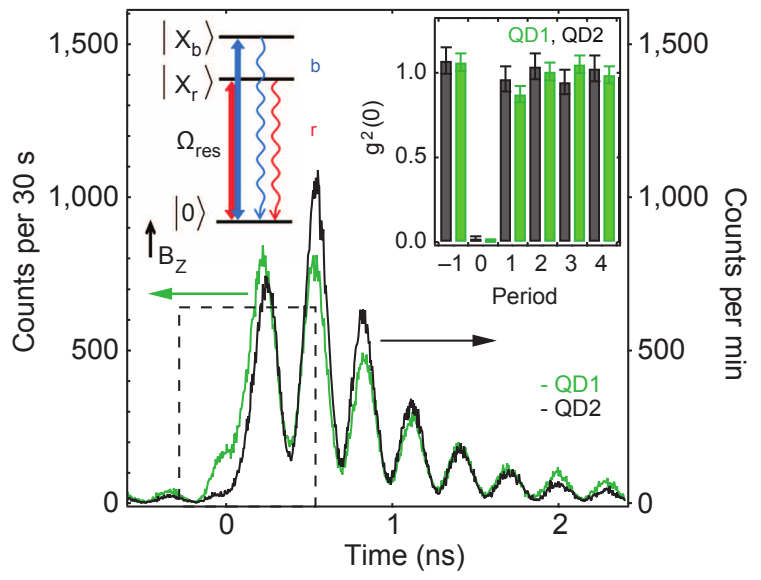

Fig. 1.5. Time resolved resonance fluorescence counts for QD1 (green) and QD2 (black). A $0.8 \mathrm{~ns}$ two-colour laser pulse (dashed line) that is resonant with both excitonic transitions excites the QD (left inset). Subsequent spontaneous emission generates a single photon in a superposition of $\omega_{r}$ and $\omega_{b}$. The matched oscillations of the emission from the two QDs at $\omega_{b}-\omega_{r}=3.45 \mathrm{GHz}$ indicate that the generated photonic qubit states are nearly identical. The right inset shows photon correlation $\left(g^{(2)}\right)$ using the photons emitted after the excitation pulse is turned off, such that the likelihood of two or more photon detection events is very small

drive $\left|X_{r}\right\rangle$ and $\left|X_{b}\right\rangle$ simultaneously. The amplitude of the modulation signal is set such that the central (carrier) peak at the laser frequency is completely suppressed. In this way, most of the laser power is carried by the frequencies $\omega_{r}$ and $\omega_{b}$. Two orthogonal polarizers on the excitation and collection paths of the $\mathrm{QD}$, set at $|H+V\rangle$ and $|H-V\rangle$, respectively, are used to suppress the laser background. Upon spontaneous emission at $t_{e}$, the generated photon is in the state $\left|\psi_{p}\right\rangle=\left(e^{i \Delta t_{e} / 2} e^{i \phi_{L}}\left|\omega_{b}\right\rangle+e^{-i \Delta t_{e} / 2} e^{-i \phi_{L}}\left|\omega_{r}\right\rangle\right) / \sqrt{2}$, where $\Delta=\omega_{b}-\omega_{r}$ and $\phi_{L}$ is the tunable relative phase imprinted by phase modulation.

The detection of the photonic frequency qubit can be implemented with a detector having time jitter smaller than $1 / \Delta$. Upon absorption of a photon, the qubit is projected onto the state $\left|\psi_{m}\right\rangle=\left(\left|\omega_{b}\right\rangle+\left|\omega_{r}\right\rangle\right) / \sqrt{2}$; the probability that a photon is registered is then $\left|\left\langle\psi_{m} \mid \psi_{p}\right\rangle\right|^{2}=\left(1+\cos \left(2 \phi_{L}+\Delta t_{e}\right)\right) / 2$. With $\phi_{L}$ and $\Delta$ fixed, the counts show an oscillation as a function of the photon emission time $t_{e}$, which can be deduced from the corresponding photon detection time $t_{d}$. The result is a temporal beat signal stemming from an interference of the two partial waves of the frequency qubit on the singlephoton detector. The measurement of a photonic frequency qubit is depicted in Fig. 1.5 for two QDs (QD1 and QD2): the beats of $\omega_{r}$ and $\omega_{b}$ demonstrate that the single photon is in a coherent superposition of two frequencies. The finite visibility of the interference of the two frequency components stems predominantly from the jitter of the detector. The single photon character of the 
photonic qubit is verified by the vanishing $g^{(2)}$ (see section 1.2 of Chap. 1) as shown in the inset of Fig. 1.5.

\subsubsection{Indistinguishability of the photonic qubits}

While indistinguishability of consecutive photons emitted by a single QD have been extensively investigated, reaching high values above $99 \%$ visibility $[12,27,28]$, what is of interest for the implementation of protocols interconnecting remote nodes is the indistinguishability of photons emitted by different emitters, that are subject to independent fluctuations and possibly slightly different characteristics (e. g. lifetime or centre frequency). In the following we present a characterization of the indistinguishability of two photonic qubits emitted by two different QDs using two-photon interference in a HongOu-Mandel (HOM) set-up [29]. The photonic frequency qubits are generated by two QDs (QD1 and QD2) placed in separate cryostats. Obtaining pairs of QDs having identical emission wavelength is challenging since self-assembled QD wavelength distribution typically spreads over a few tens of nanometers, which is about four orders of magnitude larger than the linewidth. Although the quantum confined Stark effect allows to tune the resonance to some extend (typically 10 to $30 \mathrm{GHz}$ ) it is necessary to have at one's disposal a large number of dots in order to spectrally select a matching pair from photoluminescence measurements. Once a pair of QDs with nearly identical PL emission wavelengths is found, local gate voltages applied separately to the two QDs are used to fine tune their transition frequencies into resonance. To ensure that both $\omega_{r}$ and $\omega_{b}$ of the two QDs are identical, we additionally apply local magnetic fields.

Fig. 1.6 shows the time-resolved coincidences between the two output ports of the HOM interferometer as a function of the time delay between the photon detection times, when the two input photons have either identical of orthogonal polarizations. Our measurements reveal that the visibility is $V=\left(C_{\perp}-C_{/ /}\right) / C_{\perp}=80.2 \pm 2.9 \%$, when we consider the photon emission after the laser pulse in the time window [0.8 ns, $2 \mathrm{~ns}]$. Here, $C_{\perp}\left(C_{/ /}\right)$is the total counts in the central peak with orthogonal (parallel) polarization for the input modes of the beam splitter. When the two input photons are in different periods or if they have orthogonal polarizations, then two-photon interference is absent: the observed beat signal in those cases originate exclusively from single-photon interference. With a retro-reflecting prism in one of the arms of the interferometer, we introduce a half-period time delay $(t=\pi / \Delta)$ for one of the pulses, which also renders the two photonic qubits distinguishable (Fig. 1.6b). For any given arrival time at the beam splitter, such a delay ensures that the relative phase between the two frequency components of the two single photons differ by $\pi$. In this case, the observed beat notes around the center period near zero delay time stem from an interference between the two frequency qubits $\left(\left|\omega_{b}\right\rangle+e^{i \theta}\left|\omega_{r}\right\rangle\right) / \sqrt{2}$ and $\left(\left|\omega_{b}\right\rangle-e^{i \theta}\left|\omega_{r}\right\rangle\right) / \sqrt{2}$. The sup- 


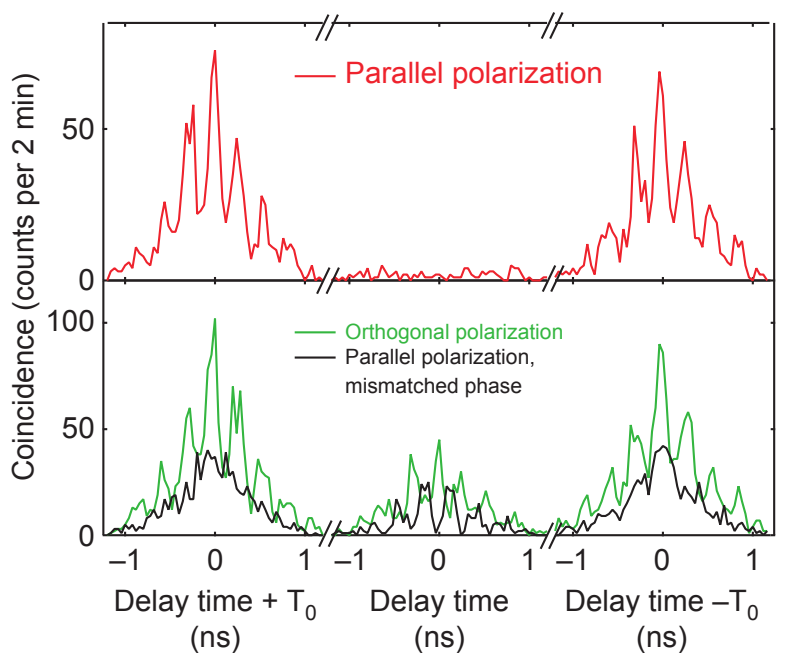

Fig. 1.6. The photonics qubits depicted in Sect. 1.3.1 are incident on a beam splitter. Coincidence counts on the two arms of the beam splitter are plotted as a function of the delay between the recorded photon arrival times. $T_{0}$ is the pulse repetition time of $13.1 \mathrm{~ns}$

pression of the beat signal in different periods stems from a superposition of two $\pi$-phase-shifted single-photon interference patterns.

\subsection{Photon to spin teleportation}

The generation of spin-photon entangled pairs (Sect. 1.2) together with the indistinguishability of single photons emitted by remote QDs (Sect. 1.3) opens the way to experimental realization of long distance quantum communication protocols. Of particular interest is the teleportation from a propagating qubit to a stationary qubit [30,31], that not only constitutes a versatile quantum-state-transfer method but can also serve as a quantum computational primitive [32-34]. In this section we focus on the recent demonstration of teleportation from a photonic frequency qubit to a QD spin qubit [35].

\subsubsection{Set-up and protocol}

In this experiment, the photonic frequency qubit to be teleported is brought into one arm of a Hong-Ou-Mandel (HOM) interferometer, while the other arm is fed with the photonic part of an entangled spin-photon pair of the form $|\Psi\rangle=\left(|\downarrow\rangle\left|\omega_{b}\right\rangle-|\uparrow\rangle\left|\omega_{r}\right\rangle\right) / \sqrt{2}$, generated as described in Sect. 1.2. The state of the coupled system consisting of two photons in modes $A$ and $B$ and the QD spin prior to the beam splitter is 


$$
|\Psi\rangle=\frac{1}{\sqrt{2}}\left(\alpha\left|\omega_{b}\right\rangle_{A}+\left|\beta \omega_{r}\right\rangle_{A}\right) \otimes\left(|\downarrow\rangle\left|\omega_{r}\right\rangle_{B}-|\uparrow\rangle\left|\omega_{b}\right\rangle_{B}\right)
$$

Provided that the single-photon pulses have identical spatiotemporal profiles, a coincidence detection at the output of the interferometer heralds successful teleportation. Indeed if the photons in modes $A$ and $B$ are indistinguishable in every aspect but their internal (frequency/colour) state, the only possibility for a simultaneous coincidence detection at the output of the HOM interferometer is to have the input two-photon state in $\left|\varphi_{S}\right\rangle=\left(\left|\omega_{b}\right\rangle_{A}\left|\omega_{r}\right\rangle_{B}-\left|\omega_{r}\right\rangle_{A}\left|\omega_{b}\right\rangle_{B}\right) / \sqrt{2}$. Therefore, detection of a coincidence projects the input photonic state (in modes $A$ and $B$ ) to $\left|\varphi_{S}\right\rangle$.

As mentioned in Sect. 1.2, the entangled state is affected by decoherence of the QD spin at a timescale $T_{2}^{*} \sim 1$ ns. To ensure that the electron spin coherence is intact for a longer time period, we introduce an optical spin-echo sequence that removes the effect of static, but random, Overhauser field, as described in Sect. 1.2.3. The electron spin is still subject to decoherence but now at a timescale that is commonly referred to as spin-echo coherence time $T_{2}$, such that $T_{2} \gg T_{2}^{*}$.

The photonic qubit that is coupled to the mode $A$ is generated by the neutral QD3, whose exciton transition energy is nearly identical to that of the QD2 trion. Local electric and magnetic fields ensure that both $\omega_{r}$ and $\omega_{b}$ are identical for both dots. We focus on photons emitted in a 800 ps long time interval, slightly longer than the 650 ps QD lifetime, and observe coincidences at the outputs of the HOM interferometer. The spin state corresponding to this measurement outcome is $\left\langle\varphi_{S} \mid \Psi\right\rangle=\alpha|\uparrow\rangle+\beta|\downarrow\rangle$, as it can be verified from eq. 1.5 .

\subsubsection{Classical correlations}

Experimental verification of teleportation is based on three-fold coincidence detection of photons at the two output modes of the HOM interferometer, together with a photon detection during the spin-measurement/preparation pulse (Fig. 1.7a). The spin population in the $|\uparrow\rangle$ state before the start of the spin-measurement pulse determines the probability that resonantly excited QD scatters one or more photons. After a few optical cycles, the spin is pumped to the $|\downarrow\rangle$ state and the QD resonance fluorescence ceases.

In order to demonstrate classical correlations between the colour of the photon to be teleported and the final spin state, we use an input photon that is prepared either in $\left|\omega_{r}\right\rangle_{A}$ or $\left|\omega_{b}\right\rangle_{A}$ and measure the spin state projected to either $|\uparrow\rangle$ or $|\downarrow\rangle$. For a mode $A$ photon in $\left|\omega_{r}\right\rangle_{A}$, a three-fold coincidence projects the photon in mode $B$ onto $\left|\omega_{b}\right\rangle_{B}$ and the spin onto $|\uparrow\rangle$. Figure $1.7 \mathrm{~b}$ shows that the same period (Period 0 ) three-fold coincidences corresponding to a spin measurement in $|\uparrow\rangle$ are a factor $\sim 4$ larger than those corresponding to $|\downarrow\rangle$. By using an input photon in state $\left|\omega_{b}\right\rangle_{A}$, the three-fold coincidences in Period 0 show that detecting the spin in $|\downarrow\rangle$ is now $\sim 4$ times more likely 

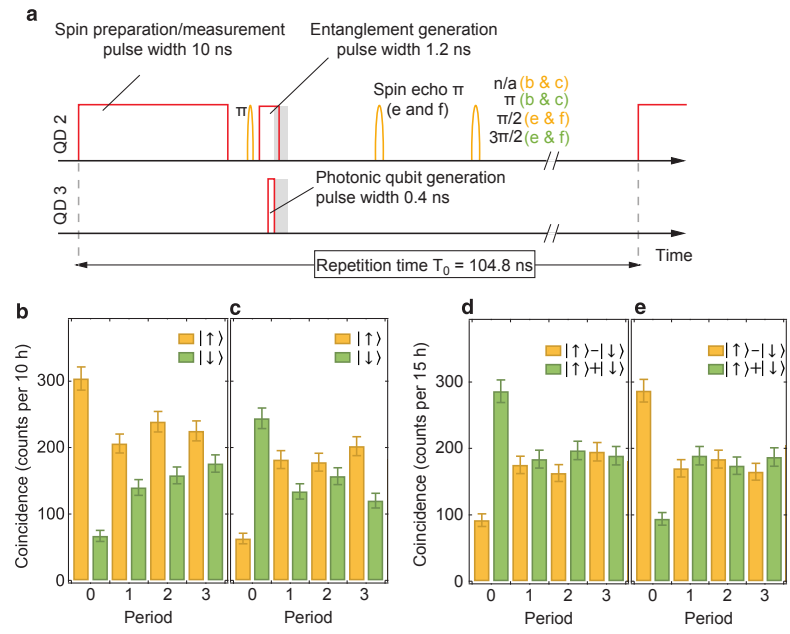

Fig. 1.7. Demonstration of quantum teleportation. a The experimental teleportation pulse sequence. The pulses applied to QD2 for entanglement generation are the same as in Fig. 1.3 with an additional spin echo $\pi$ pulse. In the spin measurement stage, different combinations of spin rotation pulses are used. For QD3, 400 ps excitation pulses are used for generating the input photonic qubit. For teleportation, we use the coincidences at the two outputs of the HOM interferometer in a $800 \mathrm{ps}$ long time-interval (labelled with the grey box). b,c Teleportation with input qubit $\left|\omega_{r}\right\rangle(\mathrm{b})$ or $\left|\omega_{b}\right\rangle$ (c). The plots show three-fold coincidence counts between the two output arms of the beam splitter and a photon detection during the following spin measurement pulse $($ Period $=0)$ or a later pulse period (Period $>0)$. The green and yellow columns represent different spin measurement basis. An enhanced probability for the spin state $|\uparrow\rangle(|\downarrow\rangle)$ as well as a decreased probability for $|\downarrow\rangle(|\uparrow\rangle)$ is observed when the photonic qubit was initially prepared in $\left|\omega_{r}\right\rangle\left(\left|\omega_{b}\right\rangle\right)$. d,e Same as b,c but with different input qubit $\left|\omega_{r}\right\rangle+\left|\omega_{b}\right\rangle$ (d) or $\left|\omega_{r}\right\rangle-\left|\omega_{b}\right\rangle$ (e). A spin-echo $\pi$-pulse is applied to prolong the spin coherence time

than detecting it in $|\uparrow\rangle$ (Fig. 1.7c), in accordance with the predictions of the teleportation protocol. From these measurements, we obtain the teleported state fidelities $0.79 \pm 0.1(0.82 \pm 0.09)$ for $\left|\omega_{r}\right\rangle_{A}\left(\left|\omega_{b}\right\rangle_{A}\right)$, where the fidelity for each teleported state is calculated as the overlap of the ideal electron spin state after the completion of the protocol with the corresponding experimental outcome for the spin state.

The different period three-fold coincidences are obtained by correlating the coincidence events for photons at the output of the HOM interferometer generated within the same period, with a spin measurement in the subsequent period. The corresponding coincidences also show a difference between the spin-up and spin-down populations, but this difference is due to a steadystate population difference between the two spin states and is not affected by the photonic qubit state. 


\subsubsection{Quantum correlations}

Demonstration of quantum teleportation requires that coherences in the photonic superposition state at the input mode $A$ are faithfully transferred onto the spin state. To verify this, we prepare the single photon in mode $A$ in either $\left|\omega_{r}\right\rangle_{A}+\left|\omega_{b}\right\rangle_{A}$ or $\left|\omega_{r}\right\rangle_{A}-\left|\omega_{b}\right\rangle_{A}$. As the propagation time of the photons onto the superconducting-single-photon detector (SSPD) is about $11 \mathrm{~ns}$, we introduce a spin-echo pulse sequence to ensure that the spin measurement is carried out only after the coincidence detection at the output of the HOM interferometer. The three-fold coincidences now indicate an enhanced probability for detection of the spin in state $|\uparrow\rangle+|\downarrow\rangle$ for an input photon in $\left|\omega_{r}\right\rangle_{A}+\left|\omega_{b}\right\rangle_{A}$ (Fig. 1.7d) and $|\uparrow\rangle-|\downarrow\rangle$ for an input photon in $\left|\omega_{r}\right\rangle_{A}-\left|\omega_{b}\right\rangle_{A}$ (Fig. 1.7e). From these measurements, we obtain the teleported state fidelities $0.76 \pm 0.03(0.75 \pm 0.03)$ for $\left|\omega_{r}\right\rangle_{A}+\left|\omega_{b}\right\rangle_{A}\left(\left|\omega_{r}\right\rangle_{A}-\left|\omega_{b}\right\rangle_{A}\right)$.

The measured teleported state fidelities are primarily limited by the small mismatch between the temporal pulse shapes and the spatial overlap profiles of the two interfering photons, as well as the finite spin-photon entanglement fidelity stemming from hyperfine interaction-mediated electron spin decoherence. Unlike measurements of spin-photon entanglement fidelity (Sect. 1.2), the experimentally determined teleportation fidelity is independent of the detector jitter. Prolongation of the spin-echo time beyond $25 \mathrm{~ns}$ in our experiments is limited by the linearly coupled Overhauser field components along the electron spin quantisation direction [39]. The relatively short timescales in which teleportation can be observed for QD2 limits possible applications of teleportation where longer memory times are needed. We note that at higher magnetic fields spin $T_{2}$ coherence times exceeding $1 \mu \mathrm{s}$ have been reported for single InAs QDs [5,39].

\subsection{Distant entanglement generation protocol}

Generation of distant entanglement between two distant QD spins can be thought as a natural extension of the teleportation experiment, where the photonic qubit is replaced by a photon that is entangled with another QD spin, thus realizing the so-called Simon-Irvine protocol [40]. A two-fold coincidence detection at the output of the HOM interferometer would herald successful generation of entanglement between the two distant spins. Entanglement generation between two distant quantum memories based on such scheme has been demonstrated in several physical systems [41,42].

The major obstacle to its realization as such with self-assembled QDs in Voigt geometry is the lack of efficient spin measurement: to verify entanglement one would need to measure both QD spins in addition to the two-fold coincidence heralding entanglement, leading to four-fold coincidence whose rate with typical collection efficiencies would be too low for a realistic implementation. 
It is however possible to herald entanglement with a single detection event of spin-flip Raman scattering, provided that it is impossible to determine the source of the detected photon [43]. Such a scheme has been previously realized with trapped ions [44] and more recently with QD hole spins [45]. In the following we will focus on the latter realization. Unlike the previously described experiments (sections 1.2 to 1.4), heralded entanglement generation has been realized with hole spin qubits, that have a longer $T_{2}^{*}$ coherence time with comparison to the electron spin (see references [46-49] and section 1.6). In the present case the holes are optically injected by resonant driving of the neutral exciton followed by tunelling of the electron to the back contact, leaving behind a single hole (see Sect. 1.5.2 of Chap. 10) as illustrated in Fig. 1.8, which depicts the relevant energy-level diagram as well as the allowed optical transitions for single-hole charged QDs in Voigt geometry. The initial states of the optical transitions in the single-hole charged regime are metastable states identified by the orientation of the heavy-hole pseudo-spin, with $|\Uparrow\rangle$ $(|\Downarrow\rangle)$ denoting $+3 / 2(-3 / 2)$ hole angular momentum projection. Presence of $B_{x} \neq 0$ yields a finite splitting of the pseudo-spin states due to heavy-light hole mixing [38]. As in the previously described case of a negatively charged QD, spontaneous emission of a $V(H)$ polarized photon at frequency $\omega_{\text {blue }}$ $\left(\omega_{\text {diag } 1}\right)$ from the trion state $\left|T_{b}\right\rangle$ at rate $\Gamma / 2$ brings the QD back into the $|\Downarrow\rangle$ $(|\Uparrow\rangle)$ state. Due to these selection rules, addressing any of the four allowed transitions with a single laser will efficiently transfer the spin population into the opposite ground state within $\sim 10 \mathrm{~ns}$.

Figure 1.9 depicts the experimental set-up where two QDs separated by 5 metres are resonantly driven by weak $3.2 \mathrm{~ns}$ long pulses from a Ti:Sapphire laser, termed the entanglement laser. Additional diode laser pulses ensure that each QD is optically charged with a single excess heavy-hole and that the hole pseudo-spin is prepared in the requisite state. Since the intensity of entanglement laser is chosen to be well below saturation, the ensuing optical transitions lead to either $V$-polarized Rayleigh scattering or $H$-polarized Raman scattering (see sections 1.2 and 1.4.3 of Chap. 3 for an extensive description of light scattering in the low power regime).

The light propagation time from the first beam splitter (BS1) to both dots, as well as from the dots to the second beam splitter (BS2) are rendered nearly identical, such that the photons scattered by the two dots during a single entanglement laser pulse reach the second beam splitter at the same time. When both QDs are initially prepared in the $|\Downarrow\rangle$ state, the simultaneous weak excitation of the blue transitions will lead to either a Raman or Rayleigh scattering event with a probability $\varepsilon^{2} \ll 1$, leaving the system in the state

$$
\begin{aligned}
|\Psi\rangle_{12}= & \frac{1}{\sqrt{2}}\left[|\Downarrow, 0\rangle+\varepsilon e^{-i \theta_{1}}\left(\left|\Uparrow, 1_{d 1, H}\right\rangle+\left|\Downarrow, 1_{b, V}\right\rangle\right)\right]_{Q D 1} \\
& \otimes\left[|\Downarrow, 0\rangle+\varepsilon e^{-i \theta_{2}}\left(\left|\Uparrow, 1_{d 1, H}\right\rangle+\left|\Downarrow, 1_{b, V}\right\rangle\right)\right]_{Q D 2} .
\end{aligned}
$$

where $\left|1_{b, H}\right\rangle$ refers to a single $H$-polarized photon with centre frequency $\omega_{b l u e}$ and $\left|1_{d 1, V}\right\rangle$ refers to a single $V$-polarized photon with centre frequency $\omega_{\text {diag } 1}$. 


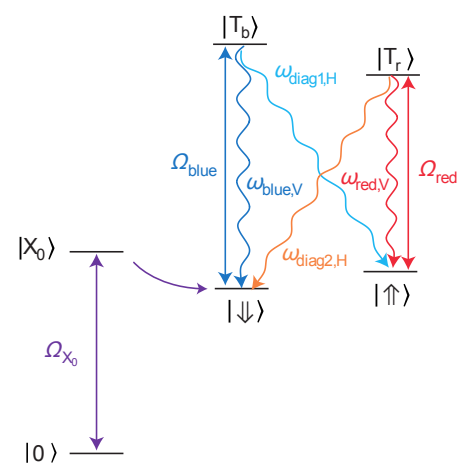

Fig. 1.8. Energy level diagram of a single QD. Upon excitation of the neutral exciton $\left(\left|X^{0}\right\rangle\right)$ state, the electron can tunnel out, leaving behind a single hole. Application of a finite magnetic field gives rise to spin-dependent optical selection rules with four allowed transitions of identical oscillator strength

To ensure that a click in one of the single-photon detectors stems from Raman scattering we use polarizers, transmission gratings and Fabry-Pérot filters. In this case, detection of a single (Raman) photon projects the composite system wave-function onto the maximally entangled state

$$
|\Psi\rangle_{12}=\frac{1}{\sqrt{2}}\left[|\Uparrow, \Downarrow\rangle+e^{-i \theta}|\Downarrow, \Uparrow\rangle\right]
$$

in the limit where two photon scattering probability $\varepsilon^{4}$ is vanishingly small. Provided that the Zeeman splitting in the two QDs are rendered identical, the relative phase $\theta=\theta_{2}-\theta_{1}$ is time-independent and is primarily determined by the optical path length difference between the two arms from BS1 to BS2 (Fig. 1.9a).

The entanglement generation scheme we use relies crucially on the indistinguishability of the photons emitted by two remote QDs (QD1 and QD2) such that "which-path" information is not available in the single-photon interferometer depicted in Fig. 1.9a. The indistinguishability of the Raman scattered photons is characterized by a Hong-Ou-Mandel experiment (see Sect. 1.3). The associated interference visibility deduced from this measurement is $91 \pm 6 \%$ guaranteeing that no substantial "which-path" information is conveyed by Raman photons.

For the protocol we implement, it is essential that the QD spins remain coherent during the time it takes for the heralding process to be completed. In our scheme the latter is determined predominantly by the $21.7 \mathrm{~ns}$ propagation time from the QDs to the single-photon detectors. In the next section, we show how Raman scattering can be used to demonstrate that the hole pseudo-spin retains its coherence on this timescale. 


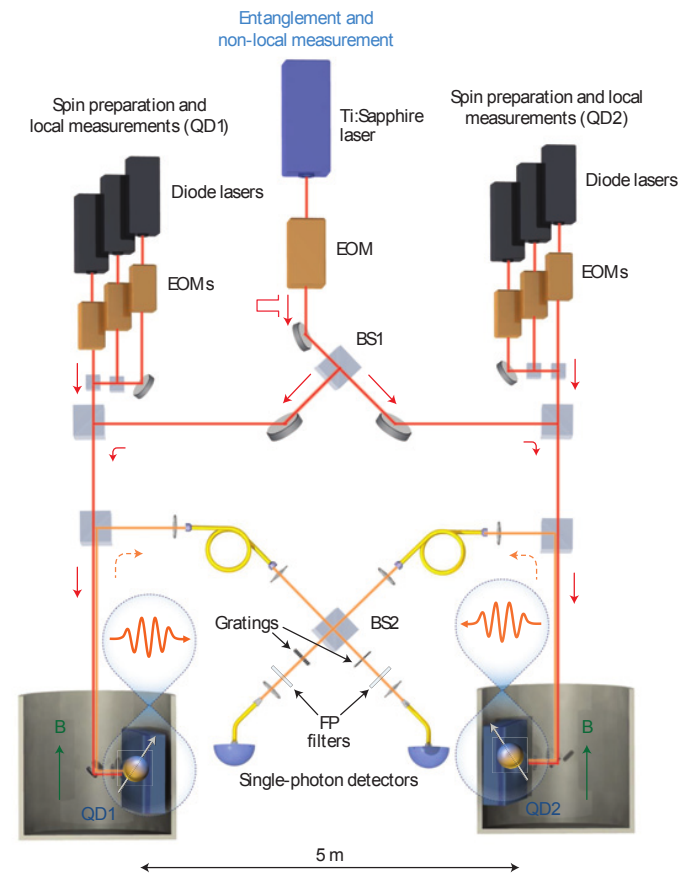

Fig. 1.9. Two bath cryostats separated by $5 \mathrm{~m}$ host QD samples in Voigt geometry. The QDs can be addressed by diode lasers (in black) for local state preparation and readout, and by a Ti:Sapphire laser (in blue) for entanglement generation and non-local measurement. EOM stands for electro-optic modulator

\subsection{Interference of Raman scattering for hole coherence measurement}

\subsubsection{Optically injected holes as coherent spin qubits}

The $T_{2}^{*}$ coherence time of the electron spin in self-assembled QDs is short, typically about $1 \mathrm{~ns}$. Such short value originates in hyperfine interaction with the fluctuating bath of nuclear spins, as discussed in Sect. 1.2.3 and in Sect. 4 of Chap. 9. On the other hand, the coherence time of the QD hole pseudo-spin in Voigt geometry has been found to exceed this value by one to two orders of magnitude, thanks to the $p$-type Bloch part of the hole wavefunction that leads to a vanishing Fermi contact term of the hyperfine interaction. The coherence time is then limited by charge fluctuations, hence being inversely proportional to the external magnetic field [37] and susceptible to vary from sample to sample. We refer the reader to the section 5 of Chap. 9 for an extensive characterisation of the hole dephasing mechanisms.

Ramsey interferometry with hole spins has been used by several groups to demonstrate values of hole $T_{2}^{*}$ between $2.3 \mathrm{~ns}[47]$ and $20 \mathrm{~ns}[48,49]$. Coherent 

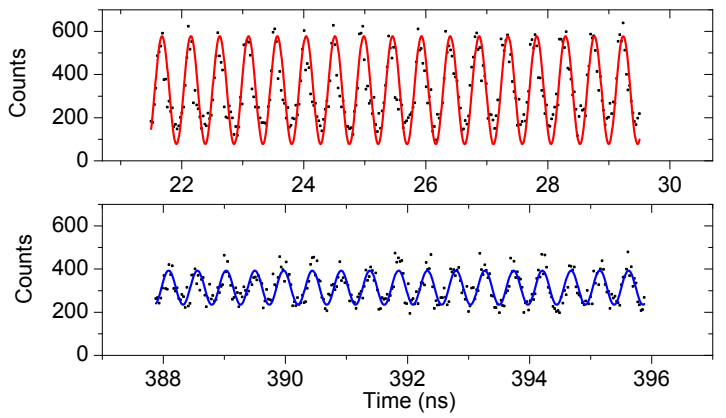

Fig. 1.10. Black squares: Ramsey interferometry performed on holes spins, revealing oscillations up to $\sim 400$ ns delay. Plain curves: sine fitting of the data.

population trapping experiments have suggested even longer coherence times at low magnetic field, up to a few hundreds of ns [46]. Ramsey interferometry performed on the sample used in Refs $[21,35,36]$ confirms such high values, indicating a coherence time of $\sim 250 \mathrm{~ns}$ for the optically injected holes at $B=1 \mathrm{~T}$ as it can be seen on Fig. 1.10.

\subsubsection{Measurement of spin coherence time using Raman scattering}

Ramsey interferometry discussed in the previous subsection is the most usual technique to characterize the $T_{2}^{*}$ coherence time of electrons and hole spins in QDs $[47,50]$. It is however strongly affected by dynamical nuclear spin polarization effects $[49,51,52]$ and requires strong detuned $\sigma$-polarized laser pulses to implement coherent rotation of the spin to be measured. An alternative of Ramsey interferometry for spin coherence measurement is single photon interference of spin-flip Raman scattering [53].

This technique is based on the fact that first-order coherence (as defined in the section 1.2 of Chap. 1) of spin-flip Raman scattering (inelastic scattering resulting a change of the spin state - see section 1.4.3 of Chap. 3) is determined by the coherence properties of the excitation laser field and the spin coherence $[54,55]$. Therefore, measuring the coherence time of Raman scattered photons upon excitation with a monochromatic laser field is equivalent to a measurement of the spin dephasing time. It is essential to carry out Raman coherence measurements at low excitation limit well below the saturation intensity in order to ensure that spin dephasing induced by Rayleigh scattering remains weak as compared to the inherent $T_{2}^{*}$ time. Moreover, dynamical nuclear spin polarization is strongly suppressed in this regime, allowing to observe the expected Gaussian decay of the interference signal.

In reference [53], this technique is demonstrated with both an electron and a hole spin and at different time delays covering the relevant timescales to observe the Gaussian decay of the interference signal. If the decay to be observed 

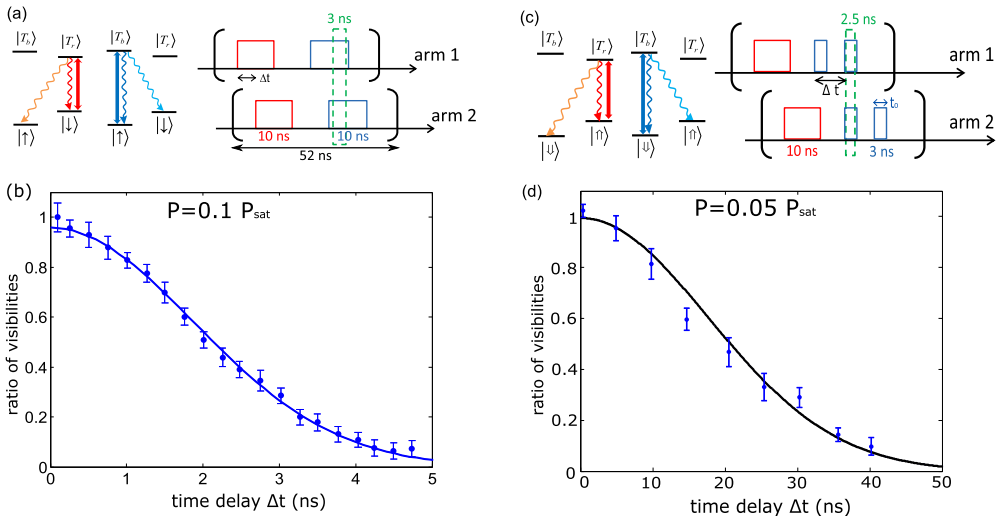

Fig. 1.11. (a) Pulse sequence used for the first-order coherence measurement of an electron spin and relevant transitions. Red square frame: $10 \mathrm{~ns}$ preparation pulse; Blue square frame: 10 ns excitation pulse; Green dashed box: 3 ns post-selected timewindow. The overall repetition rate is $52 \mathrm{~ns}$. (b)Electron spin: The ratio between the visibility of $\omega_{\text {blue }}$ photons and $\omega_{\text {diag } 1}$ photons as a function of the time delay $\Delta t$ for the excitation laser power $P=0.1 P_{\text {sat }}$. The solid curve is a Gaussian fitting of the data. (c) Pulse sequence used for the first-order coherence measurement of a hole spin and relevant transitions. Red square frame: $10 \mathrm{~ns}$ preparation pulse; blue square frames: 3 ns excitation pulse; green dashed box: 2.5 ns post-selected time-window. (d) Hole spin: The ratio between the visibility of $\omega_{\text {blue }}$ photons and $\omega_{\text {diag } 1}$ photons as a function of the time delay $\Delta t$ for the excitation laser power $P=0.05 P_{\text {sat }}$. The solid curve is a Gaussian fitting of the data

is of order nanosecond or lower, as it is the case for the electron spin, Raman scattering can be performed using a single weak laser pulse (Fig 1.11a). The loss of signal amplitude due to laser-induced decay of the spin population can be compensated by properly attenuating the transmission of the longer arm of the interferometer. The scattered photons are sent into a stabilized MachZehnder (MZ) interferometer where the visibility of the interference fringes $V_{\text {Ram }}(\Delta t)$ can be observed as a function of the time delay $\Delta t$. Rayleigh scattering interference visibility $V_{R a y}(\Delta t)$ is measured in the same conditions as a reference. Their ratio $R(\Delta t)=V_{R a m}(\Delta t) / V_{R a y}(\Delta t)$ - expressing the reduction of visibility associated with the spin decoherence - exhibits a Gaussian decay at a timescale that agrees very well with Ramsey interferometry measurements of the same QD (Fig. 1.11b).

Measuring the hole coherence time cannot be done with a single laser pulse since the signal would vanish before the spin has decohered due to spin pumping. In this case, a sequence of two pulses separated by a time delay matching the optical path length difference in the two arms of the interferometer is used (Fig $1.11 \mathrm{c}, \mathrm{d}$ ), such that spin pumping is interrupted between the two pulses, allowing to observe the decay of hole spin coherence. 
The main conclusion of this study is that in the weak excitation regime, the ratio of Raman and Rayleigh scattering interference visibilities - after a delay $\Delta t$ - directly gives the spin degree of coherence after this delay. As in the distant entanglement generation experiment, the spin has to remain coherent during the time it takes for the photon to travel from the QD to the detector (22 ns in the present case), the associated constraint on the dot pair is that both of them should display a value of $R(\Delta t=22 \mathrm{~ns})>0.5$, signifying that most of their coherence is preserved after this propagation time. In ref [45], the dots used in the experiment verify $R_{1}=58.3 \pm 3.2 \%$ and $R_{2}=53.8 \pm 6.3 \%$ at the relevant electric and magnetic field settings, which demonstrates that their coherence time is longer than what is needed for the entanglement generation protocol to take place.

\subsection{Implementation and characterisation of $z$-rotation (phase) gate}

Determination of quantum correlations rely on the ability to control the relative phase $\theta$ in the equation 1.7. The latter can be adjusted using a Vpolarized off-resonant laser field that induces different phases on the two spin states due to different magnitude of the ac-Stark effect. In the experiment of ref [45], the phase shift is performed on QD1 by applying a laser that is red-detuned by $\sim 20 \mathrm{GHz}$ from the red and $\sim 50 \mathrm{GHz}$ from the blue vertical transition (bottom right diagram of Fig. 2d). The difference in the ac-Stark shift experienced by the two transitions allows the state $|\Downarrow\rangle$ to accumulate a phase $\varphi=\Omega^{2} \tau \delta / 4 \Delta(\Delta+\delta)$, relative to $|\Uparrow\rangle$. Here, $\Omega$ is the Rabi frequency of the laser, $\tau$ the pulse duration, $\Delta$ the detuning from the red transition and $\delta=\omega_{\text {blue }}-\omega_{\text {red }}$ the energy difference between the two transitions.

In order to characterize the effect of spin-phase rotation, we performed interferometric measurements similar to those described Sect. 1.6, with the optical path-length difference kept constant. A 4 ns-long detuned laser pulse is applied in between the two weak excitation pulses (Fig. 1.12a). By varying the laser power from 0 to $\sim 2 \mu \mathrm{W}$, we change the relative phase of the two spin states and thus the relative phase of the Raman scattering amplitude before and after the pulse that induces the spin-state dependent ac-Stark shift. The oscillations in the count rate as a function of the laser power (Fig. 1.12c, black dots) unequivocally demonstrate single pseudo-spin rotation about the $z$ axis of the Bloch sphere. The red curve in Fig. 1.12c is a sinusoidal fit to the data, showing that no sizeable loss of visibility is observed for spin rotation up to $4 \pi$.

This single qubit phase gate, together with previously demonstrated spin $x$-rotation using picosecond pulses [9], provides a full control of the spin state over the whole Bloch sphere in the rotating frame. Moreover the single-qubitphase characterization scheme provides a general way to measure phase modifications of a single qubit. 
(a)

(b)
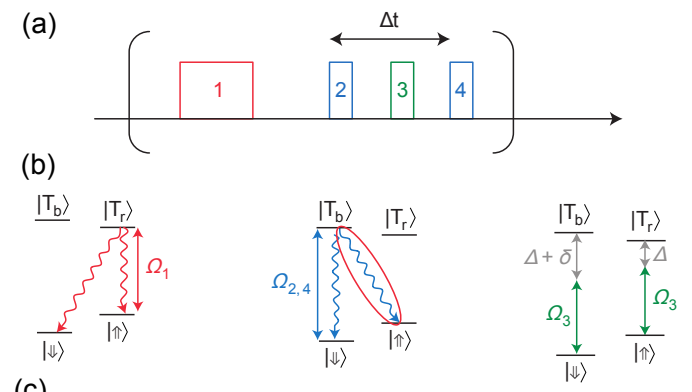

(c)

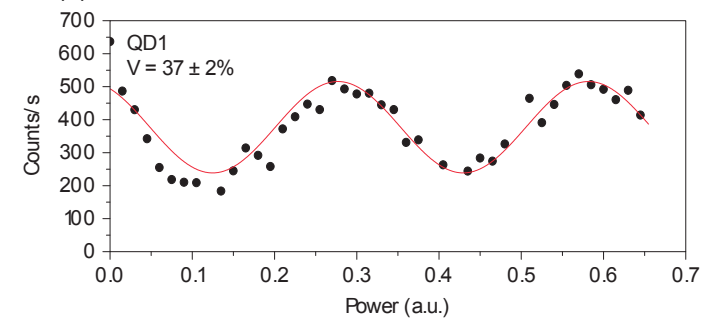

Fig. 1.12. (a) Pulse sequence used to demonstrate pseudo-spin rotation about the $z$ axis of the Bloch sphere: we first apply a pulse of frequency $\omega_{\text {red }}$ to spin pump into the $|\Downarrow\rangle$ state (pulse 1). We then apply two successive weak pulses (2 and 4) at frequency $\omega_{b l u e}$. The time offset of the two pulses approximately matches the path length difference of the MZ interferometer. An additional detuned laser pulse of $4 \mathrm{~ns}$ (pulse 3 ) is inserted in between the two pulses at $\omega_{\text {blue }}(2$ and 4 ). Light scattered during the pulse 2 and 4 interfere at the second beamsplitter. (b) Corresponding energy diagrams. (c) black dots: count rate of the output detector, as a function of the detuned laser power, demonstrating control of the pseudo-spin phase. Red curve: fit to the data. The error bars of the visibilities correspond to one standard deviation

\subsection{Experimental verification of entanglement}

Our entanglement verification scheme is based on the fact that only certain components of the generated state density matrix enter into the estimation of the state fidelity to a maximally entangled state $\left|\psi^{+}\right\rangle$, defined as $F=\left\langle\psi^{+}|\rho| \psi^{+}\right\rangle=\frac{1}{2}\left(\rho_{\Uparrow \Downarrow, \Downarrow \Uparrow}+\rho_{\Downarrow \Uparrow, \Uparrow \Downarrow}+\rho_{\Uparrow \Downarrow, \Uparrow \Downarrow}+\rho_{\Downarrow \Uparrow, \Downarrow \Uparrow}\right)$. The diagonal components $\rho_{\Uparrow \Downarrow, \Uparrow \Downarrow}+\rho_{\Downarrow \Uparrow, \Downarrow \Uparrow}$ are estimated by the measure of conditional probabilities of spin population in the computational basis (classical correlations, Sect. 1.8.1) and the off-diagonal elements $\rho_{\Uparrow \Downarrow, \Downarrow \Uparrow}+\rho_{\Downarrow \Uparrow, \Uparrow \Downarrow}$ are measured using a novel scheme based on a spin-phase dependent non-local measurement (quantum correlations, Sect. 1.8.2). 


\subsubsection{Classical correlations}

To demonstrate classical correlations between the distant spins, we carry out local single-spin measurement in the computational basis, conditioned upon the detection of a Raman photon during the entanglement pulse. Contrarily to the experiments described in sections 1.2 and 1.4 where we address a fixed spin-to-trion transition and the presence or absence of a $\pi$-pulse determines the spin state we measure, we here take benefit from the fact that each spin state can be excited to a corresponding trion state with the same oscillator strength and the same laser polarization but using a different resonant laser wavelength. The detection of a photon during a blue (red) laser pulse thus tells with a high confidence level that the state of the spin prior to the measurement pulse was $|\Downarrow\rangle(|\Uparrow\rangle)$. In order to measure the four different spin combinations under the same experimental conditions, we alternate in a single experiment four pulses sequences, each performing one of the four requisite measurement combinations. The full pulse sequence is described in Fig. 1.13a: we first prepare the state $|\Downarrow, \Downarrow\rangle$ by spin pumping, then apply the weak entanglement laser pulse. The power used is $\sim 2 \%$ of the saturation power and the Raman photon scattering probability is $\varepsilon^{2} \sim 7 \%$. The detection of a Raman photon during this pulse heralds successful entanglement generation. We then successively measure the state of the two dots. The measurement pulses of the two dots are offset in time, allowing us to extract which-path information. These two measurements are performed close to saturation and the detection efficiencies are rendered similar. The duration of the full sequence is $4 \times 104 \mathrm{~ns}$. Figure 1.13b shows the results of the 3-fold coincidences detected during 106.5 hours of measurement. As expected, the odd parity events, where the spins of the two dots are opposite, are much more likely than the even parity events, where the two spins are found in the same state. The associated fidelity is $F_{z}=80.6 \pm 6.6 \%$.

\subsubsection{Quantum correlations}

To demonstrate quantum correlations between the two distant spins, we implement a delayed two-photon interference experiment. The key element of this approach for verifying quantum correlations is the possibility to rotate one of the spins along the $z$-axis after heralded spin entanglement is generated. Application of a detuned laser pulse on QD1, as described in Sect. 1.7, results in rotating the phase of the entangled state by $\alpha(\tau)$ so that the the entangled state becomes $\left(|\Uparrow, \Downarrow\rangle+e^{-i \theta-i \alpha(\tau)}|\Downarrow, \Uparrow\rangle\right) / \sqrt{2}$. Subsequent application of a second weak (measurement) pulse, that is identical in intensity and duration to the entanglement pulse, on both QDs simultaneously leads to

$$
\begin{aligned}
|\Psi\rangle_{12}= & \frac{1}{\sqrt{2}}\left[|\Uparrow, \Downarrow, 0\rangle+\varepsilon e^{-i \theta_{2}}\left|\Uparrow, \Uparrow, 1_{d 1, H}\right\rangle\right. \\
& \left.+e^{-i \theta-i \alpha(\tau)}\left(\varepsilon e^{-i \theta_{1}}\left|\Uparrow, \Uparrow, 1_{d 1, H}\right\rangle+|\Downarrow, \Uparrow, 0\rangle\right)\right]
\end{aligned}
$$



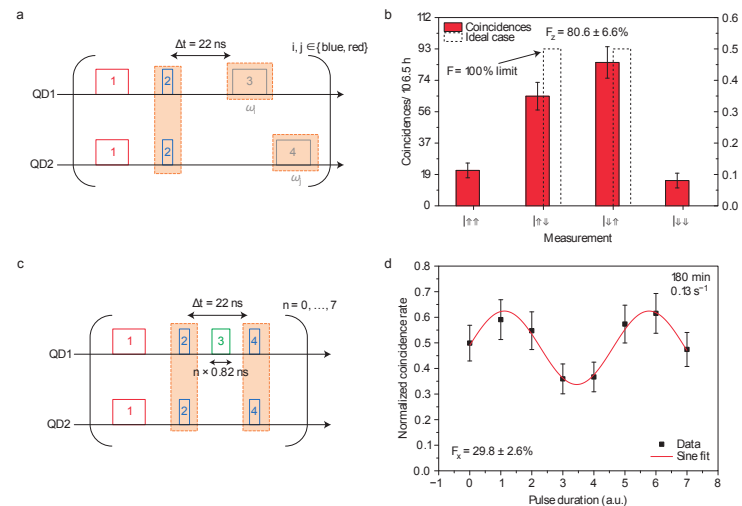

Fig. 1.13. (a) Pulse sequence used for the measurement of classical correlations between the distant spins. After spin pumping into the $\mid \Downarrow$, $\Downarrow\rangle$ state (pulse 1), a weak entanglement pulse (pulse 2) is sent simultaneously to both quantum dots (QD1 and QD2). After $22 \mathrm{~ns}$, pulse 3 measures the spin-state of QD1 and then pulse 4 measures the spin-state of QD2. The four measurement combinations are alternated. (b) Red bars: results of three-fold coincidences between a photon emitted during the entanglement pulse and a photon in each of the two measurement pulses (orange shading in Fig. 3a) obtained during a total measurement time span of $106.5 \mathrm{~h}$. The dashed bars represents the ideal limit of vanishing even parity spin state detection. The error bars represent one standard deviation deduced from poissonian statistics of the raw detection events. The measured fidelity is $F_{z}=80.6 \pm 6.6 \%$. (c) Pulse sequence used to measure quantum correlations between the distant spins. After spin pumping into the $|\Downarrow, \Downarrow\rangle$ state, a weak entanglement pulse (pulse 2) is used to drive both QDs. A detuned laser pulse (pulse 3) modifies the phase of the QD1 hole spin phase. After $22 \mathrm{~ns}$, a non-local measurement pulse is applied to both QDs. The pulse sequence is repeated for different values of the duration of the pulse 3 ranging from 0 to $8 \times 0.82 \mathrm{~ns}$, corresponding to a laser-induced QD1 spin phase rotation ranging from 0 to $3 \pi$. (d) Black dots: two-fold coincidence rate between a photon detected during the entanglement pulse and a second photon detected during the measurement pulse (orange shading in Fig. 3c), normalized by the average detection rate between photons emitted during different periods, as a function of the pulselength of pulse 3 . The error bars represent one standard deviation deduced from poissonian statistics of the raw detection events. The red curve is a sinusoidal fit to the data, yielding a visibility of $V=29.8 \pm 2.6 \%$. The deduced overall fidelity is $F=\left(F_{z}+V\right) / 2=55.2 \pm 3.5 \%$

$$
\begin{aligned}
= & \frac{\varepsilon}{\sqrt{2}} e^{-i \theta_{2}}\left(1+e^{-i \alpha(\tau)}\right)\left|\Uparrow, \Uparrow, 1_{d 1, H}\right\rangle \\
& +\frac{1}{\sqrt{2}}\left(|\Uparrow, \Downarrow, 0\rangle+e^{-i \theta-i \alpha(\tau)}|\Downarrow, \Uparrow, 0\rangle\right) .
\end{aligned}
$$

Therefore, conditioned on an initial Raman photon detection event that heralded spin-spin entanglement, the detection of a second time-delayed Raman 
photon detection probability scales as $\varepsilon^{2}\left|1+e^{-i \alpha(\tau)}\right|^{2}$. The expectation value of Raman photon detection can be shown to be

$$
\left\langle E^{(-)} E^{(+)}\right\rangle \propto 1+0.5\left\langle\sigma_{z}^{1}+\sigma_{z}^{2}\right\rangle-\left\langle\sigma_{\Downarrow \Uparrow}^{2} \sigma_{\Uparrow \Downarrow}^{1}+\sigma_{\Downarrow \Uparrow}^{1} \sigma_{\Uparrow \Downarrow}^{2}\right\rangle .
$$

The peak-to-peak contrast in $\left\langle E^{(-)} E^{(+)}\right\rangle$obtained by varying $\alpha(\tau)$ therefore gives us the magnitude of non-local quantum correlations between the two spins.

To verify the presence of quantum correlations using such a delayed two-photon interference experiment, we use the pulse sequence described in Fig. 1.13c. We once again prepare the spins in the $|\Downarrow, \Downarrow\rangle$ state by spin pumping and then apply the weak entanglement generation pulse in the same way as for the classical correlation measurement. The phase of the state is then modified by the detuned laser pulse, whose duration is changed within the pulse sequence by alternating eight patterns that differ only by the duration of this particular pulse. Eight evenly distributed durations are chosen to cover more than one full revolution. Finally the measurement pulse is simultaneously sent to both dots. The duration of the full sequence is $8 \times 52 \mathrm{~ns}$. The two-fold coincidences measured for each value of the pulse length are normalized by the uncorrelated coincidence rate obtained by measuring two photons emitted in different periods. Figure 1.13d presents data obtained during $180 \mathrm{~min}$ of measurement. The obtained ratio exhibits clear oscillations of visibility $29.6 \pm 2.8 \%$.

\subsubsection{Discussion}

Combining the results depicted in Figs. 1.13b and 1.13d, we deduce an overall fidelity of the generated entangled state of $F=55.2 \pm 3.5 \%$. Although this number is relatively modest compared to previous work based on other physical systems $[41,42,44,56,57]$, it is predominantly limited by the $T_{2}^{*}$ coherence time of the hole spins and hence could be substantially increased by either a decrease in the magnetic field [37] or introduction of dynamical decoupling [47]. The detection rate of single photons emitted during the entanglement pulse is 2300 photons per second; the latter directly yields the heralded entanglement generation rate. Such high value has been made possible by the use of a high repetition rate $\left(\Gamma_{r e p}=1.9 \times 10^{7} \mathrm{~s}^{-1}\right)$ allowed by the fast spin initialization (10 ns) together with a relatively high collection efficiency of $\sim 20 \%$ to the objective leading to an overall collection efficiency of $\sim 0.2 \%$, and could be further increased using cavity QED [10]. On the other hand, the lack of efficient spin measurement leads to a low three-fold coincidence rate of $\sim 2 / \mathrm{h}$. The scheme we presented in Sect. 1.8.2 allows verification of non-local quantum coherence using two-fold coincidence, and therefore can be performed much faster than a full state tomography. 


\subsection{Conclusion and outlook}

In this chapter we have reviewed recent demonstrations of elementary quantumdot-based quantum communication protocols, namely entanglement between a QD spin and a propagating photon, teleportation from a propagating photon to a QD spin and generation of heralded entanglement between distant QD hole spins.

When it comes to comparing these results with those previously obtained with other physical systems, one can identify a major advantage of QD-based realizations which is the possibility to obtain much higher success rates. This comes as a result of the advantages offered by semiconductor technological possibilities that allow easy integration into semiconductor structures which in turn enables both electrical and photonic control of the environment for optimized photon collection efficiency [12]. Moreover thanks to the short lifetime $(<1 \mathrm{~ns})$ of the excited states it is possible to perform state initialization within $10 \mathrm{~ns}$ and therefore operate at high repetition rates.

On the other hand, the main drawback in QD-based implementations of quantum communication protocols such as those presented in this chapter is the fact that the coherence time of QD spins is relatively short as compared to other commonly used physical system, such as atoms or NV centres. The coherence times involved in the presented experiments (both for electrons with echo and holes without echo) are of order of a few tens of nanoseconds, and the best reported spin-echo $T_{2}$ coherence times are at most of order microsecond. This limits the use of QDs for quantum communication or distributed quantum computation to about a kilometre, but should however not be a limitation for on-chip applications.

Another major difficulty comes from the inefficient spin measurement for single QDs in Voigt geometry. The use of singlet-triplet qubits in QD molecules could allow to circumvent this obstacle since it can not only prolong the coherence times [26] but also simultaneously allow for single-shot readout [58].

\section{Acknowledgements}

The authors would like to acknowledge their coworkers Emre Togan, Parisa Fallahi, Martin Kroner, Yves Delley, Javier Miguel-Sanchez and Stefan Fält for their extensive participation in the presented work.

\section{References}

1. J.I. Cirac, P. Zoller, H.J. Kimble, H. Mabuchi, Phys. Rev. Lett. 78, 3221 (1997).

2. L.M. Duan, M.D. Lukin, J.I. Cirac, P. Zoller, Nature 414, 413 (2001)

3. H.J. Kimble, Nature 453, 1023 (2008)

4. S. Ritter et al. Nature 484195 (2012)

5. D. Press et al., Nature Photon. 4, 367 (2010) 
6. A. Imamoğlu, D.D. Awschalom, G. Burkard, D.P. DiVincenzo, D. Loss, M. Sherwin, A. Small Phys. Rev. Lett. 83, 4204 (1999)

7. S.T. Yilmaz, P. Fallahi, A. Imamoğlu, Phys. Rev. Lett. 105, 033601 (2010)

8. M. Atatüre, J. Dreiser, A. Badolato, A. Högele, K. Karrai, A. Imamoğlu, Science 312, 551 (2006)

9. D. Press, T.D. Ladd, B. Zhang, Y. Yamamoto, Nature 456, 218 (2008)

10. O. Gazzano et al. Nature Comm. 4, 1425 (2013)

11. A. Högele et al. Phys. Rev. Lett. 93, 217401 (2004)

12. N. Somaschi et al. Nature Photon. 10, 340 (2016)

13. G. Reithmaier, S. Lichtmannecker, T. Reichert, P. Hasch, K. Müller, M. Bichler, R. Gross, J.J. Finley Scientific Reports 3,1901 (2013)

14. B.B. Blinov, D.L. Mohering, L.M. Duan, C. Monroe, Nature 428, 153 (2004)

15. T. Wilk, S.C. Webster, A. Kuhn, G. Rempe, Science 317, 488 (2007)

16. E. Togan et al., Nature 466, 730 (2010)

17. C. Arnold et al., Nature Comm. 6, 6236 (2015)

18. X. Xu et al., Phys. Rev. Lett. 99, 097401 (2007)

19. K. De Greve et al., Nature 491, 421 (2012)

20. J.R. Schaibley et al. Phys. Rev. Lett. 110, 167401 (2013)

21. W.B. Gao, P. Fallahi, E. Togan, J. Miguel-Sanchez, A. Imamoğlu, Nature 491, $426(2012)$

22. A. Muller et al., Phys. Rev. Lett. 99, 187402 (2007)

23. A.N. Vamivakas, Y. Zhao, C.Y. Lu, M. Atatüre, Nature Phys. 5, 198 (2009)

24. K. De Greve et al. Nature Comm. 4, 2228 (2013)

25. P.L. McMahon, K. De Greve, in Engineering the Atom-Photon Interaction, ed. by A. Predojević, M.W. Mitchell (Springer, 2015), p. 365-402

26. K.M. Weiss, J.M. Elzerman, Y.L. Delley, J. Miguel-Sanchez, A. Imamoğlu, Phys. Rev. Lett. 109, 107401 (2012)

27. C. Santori, D. Fattal, J. Vučković, G.S. Solomon, Y. Yamamoto, Nature 419, $594(2002)$

28. S. Ates, S.M. Ulrich, S. Reitzenstein, A. Löffler, A. Forchel, P. Michler, Phys. Rev. Lett. 103, 167402 (2009)

29. C.K. Hong, Z.Y. Ou, L. Mandel, Phys. Rev. Lett. 59, 2044-2046 (1987)

30. J.F. Sherson et al., Nature 443, 557 (2006)

31. Y.A. Chen et al. Nature Phys. 4, 103 (2008)

32. D. Gottesman, I.L. Chuang, Nature 402, 390 (1999)

33. E. Knill, R. Laflamme, G.J. Milburn, Nature 409, 46 (2001)

34. W.B. Gao et al. Proc. Natl Acad. Sci. 107, 20869 (2010)

35. W.B. Gao, P. Fallahi, E. Togan, A. Delteil, Y.S. Chin, J. Miguel-Sanchez, A. Imamoğlu, Nature Comm. 4, 2744 (2013)

36. A. Delteil, W.B. Gao, P. Fallahi, J. Miguel-Sanchez, A. Imamoğlu, Phys. Rev. Lett. 112, 116802 (2014)

37. J.H. Pretchel et al. Phys. Rev. B 91, 165304 (2015)

38. M. Bayer et al., Phys. Rev. B 65, 195315 (2002)

39. R. Stockill, C. Le Gall, C. Matthiesen, L. Huthmacher, E. Clarke, M. Hugues and M. Atatüre, arXiv:1512.01811 (2015)

40. C. Simon, W. Irvine, Phys. Rev. Lett. 91, 110405 (2003)

41. D.L. Moehring et al. Nature 449, 68 (2007)

42. H. Bernien et al. Nature 497, 86 (2013)

43. C. Cabrillo, J.I. Cirac, P. García-Fernández, P. Zoller, Phys. Rev. A 59, 1025 (1999) 
44. L. Slodička, G. Hétet, N. Röck, P. Schindler, M. Hennrich, R. Blatt, Phys. Rev. Lett. 110, 083603 (2013)

45. A. Delteil, Z. Sun, W. Gao, E. Togan, S. Faelt, A. Imamoğlu, Nature Phys. 12, 218 (2016)

46. D. Brunner et al. Science 325, 70 (2009)

47. K. De Greve et al. Nature Phys. 7, 872 (2011)

48. K. Greilich, S.G. Carter, D. Kim, A.S. Bracker, D. Gammon, Nature Photon. 5, 702 (2011)

49. S.G. Carter et al. Phys. Rev. B 89, 075316 (2014)

50. N. F. Ramsey, Phys. Rev. 78, 695 (1950)

51. A. Högele, M. Kroner, C. Latta, M. Claassen, I. Carusotto, C. Bulutay, and A. Imamoğlu, Phys. Rev. Lett. 108, 197403 (2012).

52. T. D. Ladd, D. Press, K. De Greve, P. L. McMahon, B. Friess, C. Schneider, M. Kamp, S. Höfling, A. Forchel, and Y. Yamamoto, Phys. Rev. Lett. 105, 107401 (2010).

53. Z. Sun, A. Delteil, S. Faelt, A. Imamoğlu, Phys. Rev. B 93, 241302(R) (2016)

54. G. Fernandez, T. Volz, R. Desbuquois, A. Badolato, and A. Imamoğlu, Phys. Rev. Lett. 103, 087406 (2009)

55. T. M. Sweeney et al., Nature Photon. 8, 442 (2014)

56. D. Hucul et al. Nature Phys. 11, 37-42 (2015)

57. J. Hofmann et al. Science 337, 72-75 (2012)

58. Y. L. Delley, M. Kroner, S. Faelt, W. Wegscheider, A. Imamoğlu, arXiv:1509.04171 



\title{
Index
}

\author{
coherence time, $8,16,19,20,26,27 \quad$ phase gate, 22 \\ correlations \\ classical, 6, 14, 24 \\ quantum, 7, 16, 24 \\ photonic qubit, 9 \\ quantum dot molecules, 27 \\ distant spin entanglement, 16, 23 \\ entanglement, 2 \\ Raman scattering, 20 \\ Ramsey interferometry, 19 \\ fidelity, 8, 16, 26 \\ selection rules, 3 \\ indistinguishability, 9, 12 \\ spin-echo, 8 \\ nuclear spins, 8 \\ teleportation, 13
}


